\title{
Thoracoabdominal aortic aneurysm repair
}

\section{Background}

An aneurysm is localized abnormal dilatation or widening of a blood vessel, at least $50 \%$ more than its normal size. The aorta is the largest blood vessel in the body, extending from the heart down through the thorax and abdomen until it divides to form the major arteries of the legs. Thoracoabdominal aortic aneurysm (TAAA) refers to an abnormal widening of the aorta involving both its thoracic and abdominal segments. Although atherosclerosis and TAAA share common risk factors and frequently co-exist, these aneurysms are primarily the result of age related degeneration and weakening of the aortic wall. Connective tissue disorders, dissections (separation of the wall layers), infections, and inflammations are the other causes.

\section{Seeking Help}

Pain is the most common symptom, but almost half do not have symptoms and many present as an incidental finding on imaging such as CT scans. It is important to diagnose and treat aneurysms as there is a risk of rupture or dissection ( 1 out of 10 cases), which are life threatening. Increasing pain, sweatiness, dizziness, fast pulse, loss of consciousness, weakness or paralysis, and shortness of breath are all symptoms of impending complications. Any of these symptoms or a family history should prompt one to seek medical help. The investigations would include a chest X-ray, echocardiogram, CT scan and/or MRI scan.

\section{Medical Management}

If the aneurysm does not meet the criteria for intervention, your doctor would continue to monitor it by serial scans and medications to treat your blood pressure (e.g., beta blockers) and / or cholesterol lowering agents (statins).

\section{Surgical Management}

Elective surgical intervention is considered when the aortic size exceeds 5 to $6 \mathrm{~cm}$ or when the rate of dilatation is more than $1 \mathrm{~cm}$ per year. Presence of connective tissue disorders, body size, and other criteria might modify the decision. TAAA repair refers to the surgical repair of such aneurysms. At present there are three operative techniques: open, endovascular and hybrid.

Open TAAA repair involves surgical removal of a section of the aorta and replacing it with an artificial graft. This is an extremely extensive and complicated operation. Usually, a large incision extending from the upper back on the left side, curving around under the shoulder-blade, around to the front of the abdomen, down to the umbilicus/or even to the pubic bone. Operating on the aorta involves clamping it in stages and hence a heart lung machine is sometimes used to preserve blood supply and nutrition to vital organs such as the spinal cord, kidneys, liver and the bowel.

Endovascular approach is a much less invasive approach. Small incisions are made to access blood vessels in the groin. A catheter is inserted and used to deploy a stent graft within the aneurysm.

Hybrid approach uses a combination of these two techniques. The choice of technique depends on the patient's physiologic reserve, co-morbidities, vascular anatomy and experience of the treating surgeon/center.

\section{Benefits}

Despite the complexity and high risk, elective operation gives good long-term results and prevents the catastrophic complications of rupture and dissection.

\section{Risks}

This kind of surgery is not without significant risks.

The most serious risks associated with TAAA repair are death, stroke, and damage to the spinal cord resulting in paraplegia. The rates of these risks vary between institutions and depend on individual patient anatomy and comorbidity. As with all surgical interventions, the potential risks need to be weighed against the benefits and discussed with your surgeon.

For more information, visit the following websites:

http://emedicine.medscape.com/article/424904-overview http://my.clevelandclinic.org/heart/disorders/aorta_marfan/ aorticaneurysm.aspx

www.mayoclinic.com/health/aortic-aneurysm http://ats.ctsnetjournals.org/cgi/content/full/74/5/S1877

doi: 10.3978/j.issn.2225-319X.2012.09.04

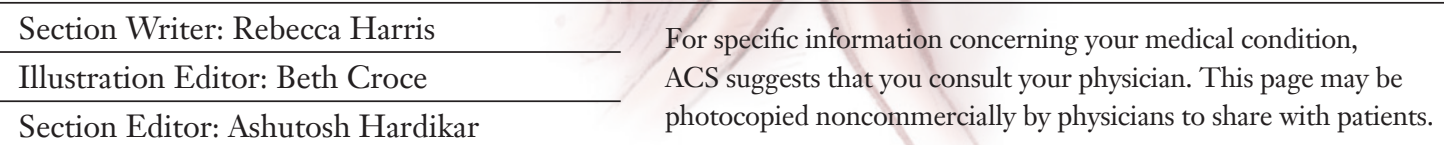

